\title{
Approximate semi-analytical solutions for the steady-state expansion of a contactor plasma
}

\author{
E Camporeale $^{1}$, E A Hogan ${ }^{2}$ and E A MacDonald ${ }^{3}$ \\ ${ }^{1}$ Center for Mathematics and Computer Science (CWI), Amsterdam, The Netherlands \\ ${ }^{2}$ University of Colorado, Boulder, CO, USA \\ ${ }^{3}$ NASA Goddard Space Flight Center, Greenbelt, MD, USA \\ E-mail: e.camporeale@cwi.nl
}

Received 26 September 2014, revised 17 December 2014

Accepted for publication 5 January 2015

Published 13 February 2015

\begin{abstract}
We study the steady-state expansion of a collisionless, electrostatic, quasi-neutral plasma plume into vacuum, with a fluid model. We analyze approximate semi-analytical solutions, that can be used in lieu of much more expensive numerical solutions. In particular, we focus on the earlier studies presented in Parks and Katz (1979 American Institute of Aeronautics, Astronautics Conf. vol 1), Korsun and Tverdokhlebova (1997 33rd Joint Prop. Conf. (Seattle, WA) AIAA-97-3065), and Ashkenazy and Fruchtman (2001 27th Int. Electric Propulsion Conf. (Pasadena, CA)). By calculating the error with respect to the numerical solution, we can judge the range of validity for each solution. Moreover, we introduce a generalization of earlier models that has a wider range of applicability, in terms of plasma injection profiles. We conclude by showing a straightforward way to extend the discussed solutions to the case of a plasma plume injected with non-null azimuthal velocity.
\end{abstract}

Keywords: self-similar solutions, plasma contactor, spacecraft charging

(Some figures may appear in colour only in the online journal)

\section{Introduction}

The modeling of the expansion of a plasma plume in the vicinity of a spacecraft has been intensively investigated in the last several years. In particular, in the context of space electric propulsion systems, such as ion or Hall thrusters, a correct characterization of the emitted plasma plume is of crucial importance to avoid interactions between the energetic particles and spacecraft surface, and thus to prevent severe damages to the spacecraft [4-7]. Similarly, in plasma contactor technology (for instance, electrodynamics tether applications), it is important to predict the shape and geometry of the plasma plume emitted by a spacecraft $[8,9]$.

Due to the large difference in density between the plasma plume and the background magnetospheric plasma, the physics of the plume expansion is often well described by the expansion of plasma in vacuum, which has also been investigated thoroughly for several different geometries $[10,11]$. The firstprinciples numerical calculation of the steady-state profiles reached by the expansion of a plasma plume in a large domain is very challenging, due to the wide separation of scales involved. For instance, the plasma Debye length can vary by several order of magnitude in a few hundred meters along the expansion trajectory. Therefore, numerical simulations are usually constrained to either the near- or the far-field expansion, i.e. to a restricted simulation domain [12-18].

A very appealing alternative to expensive numerical simulations is, of course, to seek for analytical solutions of the plume expansion. This is often done by assuming that the plasma obeys simplified fluid equations [1-3, 19-21].

In this paper we analyze and discuss the self-similar solutions presented in [1-3]. It is important to notice that the self-similar approach used in such models is different from the standard procedure followed in similarity methods described, for instance, in classical textbooks [22]. In fact, the standard approach is based on the idea of reducing the dimensionality of a set of partial differential equations, through an appropriate 
variable transformation, that can be identified by exploiting the symmetries of the system. On the other hand, the models described in this paper retain their full dimensionality, and are based on a variable transformation constructed on the principle that one variable is constant along fluid streamlines. Another crucial point to notice is that the solutions presented in [1-3] are approximate solutions. As we will show, the self-similarity assumption is indeed inconsistent with the fluid model employed and, as such, the solutions obtained do not exactly solve the initial set of equations. However, such solutions, albeit inexact, have the advantage that they can be quickly evaluated on an arbitrary large domain. For this reason, they can still be valuable, if one is able to estimate how large their error is with respect to the true solution, and, of course, if such error is reasonably small.

The aim of this paper is twofold. First, we analyze the earlier solutions of [1-3] and we measure their errors with respect to the numerical solution of the underlying fluid equations. Second, we present a generalization of such solutions, in an attempt to provide a more flexible family of solutions that can have a wider range of applicability.

The paper is organized as follows. Section 1 presents the mathematical model, the self-similarity assumption, and the resulting set of equations. In section 2, we describe the approximate solutions presented in [1-3], and we comment on their range of validity and respective errors. In section 3, we describe a new class of solutions, that is based on a generalization of previous models, and we show that they are indeed applicable to a wider class of situations. In section 4 we show how to easily extend the solutions that were derived for a plasma with no azimuthal velocity to a more general case. Finally, we draw conclusions of this study in section 5 .

\section{Mathematical model}

We study a system composed by collisionless, singly charged, ions and electrons, in a steady-state, axisymmetric configuration. We assume that electrons inertia can be neglected, quasi-neutrality holds, the plasma is electrostatic, and there is no background magnetic field. For simplicity, we assume cold ions although a polytropic equation of state for ions might be easily incorporated in the model. By employing cylindrical coordinates $(r, z, \theta)$ the ion continuity equation and the conservation of ion and electron momentum read:

$$
\begin{gathered}
\frac{\partial\left(n u_{z}\right)}{\partial z}+\frac{1}{r} \frac{\partial\left(r n u_{r}\right)}{\partial r}=0, \\
u_{z} \frac{\partial u_{r}}{\partial z}+u_{r} \frac{\partial u_{r}}{\partial r}-\frac{u_{\theta}^{2}}{r}=-\frac{\partial \phi}{\partial r}, \\
u_{z} \frac{\partial u_{z}}{\partial z}+u_{r} \frac{\partial u_{z}}{\partial r}=-\frac{\partial \phi}{\partial z}, \\
u_{z} \frac{\partial u_{\theta}}{\partial z}+u_{r} \frac{\partial u_{\theta}}{\partial r}+\frac{u_{\theta} u_{r}}{r}=0, \\
\frac{1}{n} \nabla p_{e}=\nabla \phi
\end{gathered}
$$

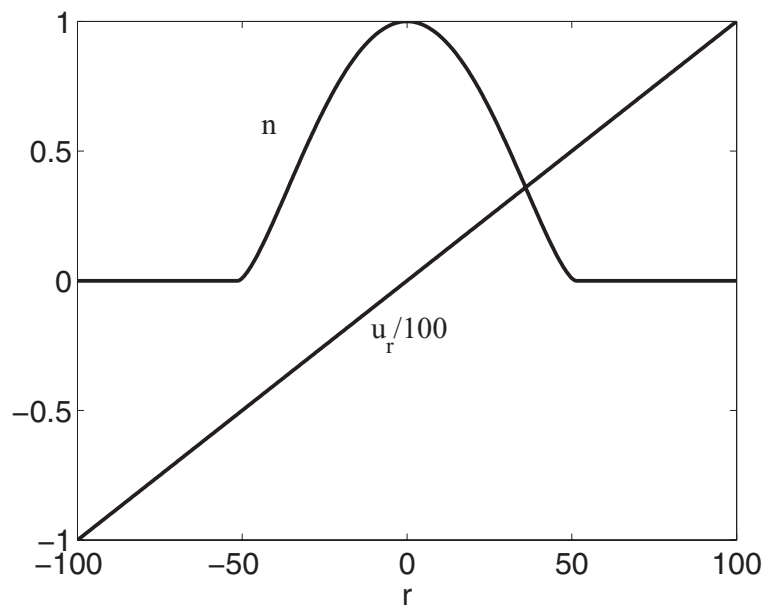

Figure 1. PK solution. Profiles for the density $n$ and the radial velocity $u_{r}$ (rescaled by a factor of 100) at the injection boundary $z=0$. In the region $r \gtrsim 50$ where the density becomes imaginary, it is artificially set equal to zero.

where the symmetry condition $\frac{\partial}{\partial \theta}=0$ has been used, $n$ is the density, $u_{z}$ and $u_{r}$ are the ion axial, radial, and azimuthal velocities, $\phi$ is the electrostatic potential, and $p_{\mathrm{e}}$ is the electron pressure. Equations (1)-(5) are complemented by a polytropic equation of state for the electrons:

$$
p_{e}=n^{\gamma}
$$

with $\gamma$ the polytropic index. Quantities have been normalized as follows: velocities to $\sqrt{T_{0} / m_{i}}$ (with $T_{0}$ a reference electron temperature and $m_{i}$ the ion mass), the electrostatic potential to $T_{0} / e$ (with $e$ the elementary charge), density to a reference density $n_{0}$, lengths to a characteristic length $R$, and pressure to $n_{0} T_{0}$. In general, the value of the polytropic index $\gamma$ depends on the degree of ionization of the plasma [23]. For instance, [7] have reported experimental results for thrusters, with $\gamma$ lower than 5/3, and [24] have used the value 1.3 for their PIC simulations. However, as shown in [23], $\gamma$ tends to the neutral gas theoretical value for a fully ionized plasma, which, for simplicity, is the case treated in this paper. Hence, for all the cases presented here $\gamma=5 / 3$, i.e. the adiabatic constant of the monoatomic perfect gas.

The model in equations (1)-(5) must be interpreted as a boundary value problem in the two-dimensional plane $(r, z)$. Since the equations involved are first-order partial differential equations, one is allowed to specify the boundary conditions at $r=0$ and $z=0$, which determine the solution in the whole domain. Also, note that the electron pressure and the electric potential can be substituted in equations (2)-(3), and post-processed after the solution for $n, u_{r}, u_{z}$ and $u_{\theta}$ has been obtained.

\subsection{Self-similar solution}

We now elucidate the procedure to seek for a self-similar solution of equations (1)-(5), extending the derivation in [25] to the case $u_{\theta} \neq 0$. First, we introduce a change of variable $(r, z) \rightarrow(\eta, z)$, with $\eta=\frac{r}{a(z)}$, and $a(z)$ an unspecified 

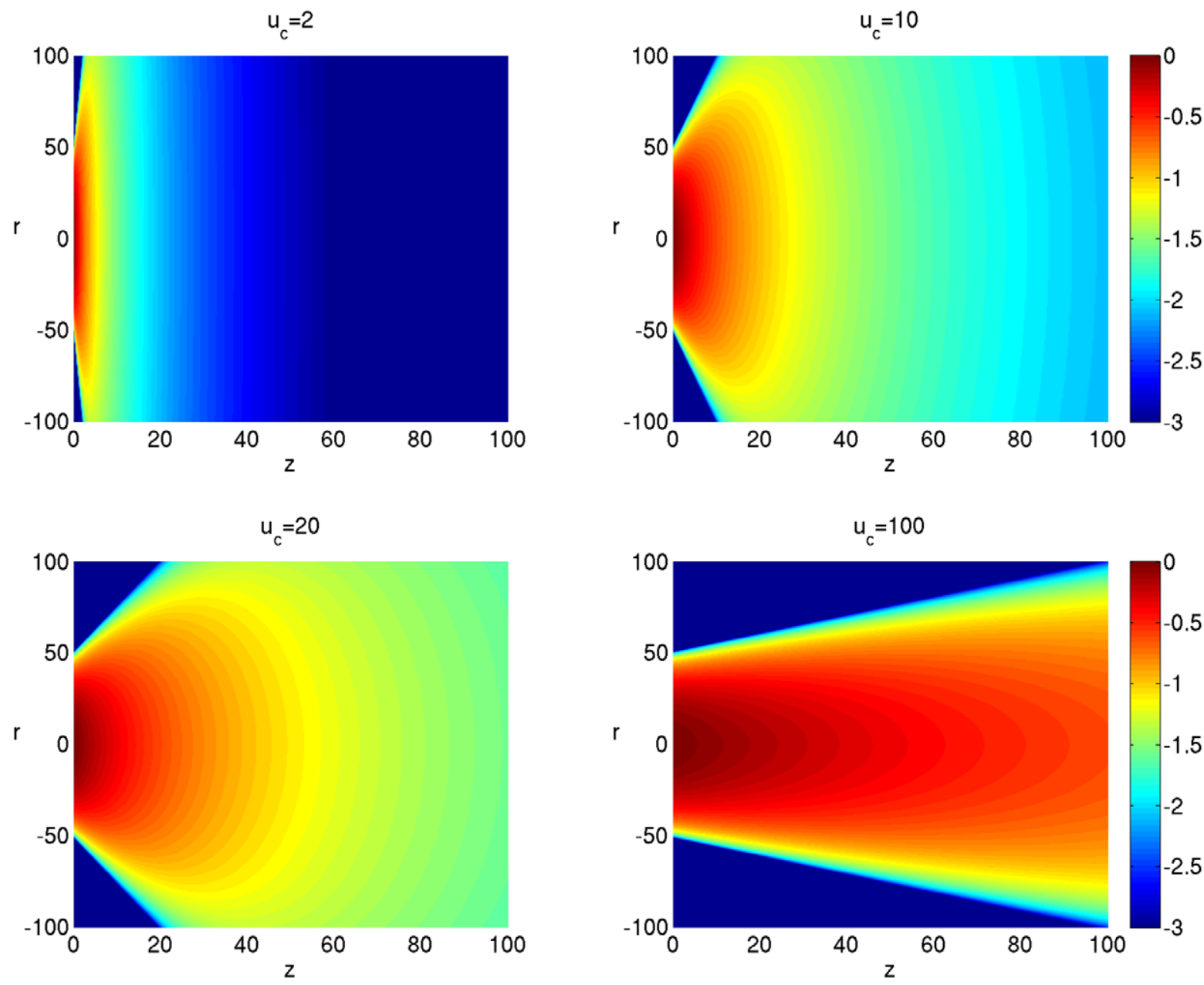

Figure 2. PK solution. Two-dimensional profiles of the density in $(z, r)$, for $u_{c}=2,10,20,100$, in logarithmic scale. The density is artificially set equal to zero in the regions where it becomes imaginary.

function. Then, we assume that the unknowns $n, u_{z}, u_{r}, u_{\theta}$ can be factorized as

$$
\begin{aligned}
n(\eta, z) & =n_{c}(z) n_{t}(\eta), \\
u_{z}(\eta, z) & =u_{c}(z) u_{t}(\eta), \\
u_{r}(\eta, z) & =\eta a^{\prime}(z) u_{z}(\eta, z), \\
u_{\theta}(r, \eta) & =\frac{\eta}{a(z)} E u_{t}(\eta),
\end{aligned}
$$

where prime indicates differentiation, and $E$ is an arbitrary constant. The definition for $u_{r}$, equation (9), follows from assuming that lines of constant $\eta$ correspond to streamlines, that is

$$
\left.\frac{\partial \eta}{\partial r}\right|_{z} u_{r}+\left.\frac{\partial \eta}{\partial z}\right|_{r} u_{z}=0
$$

As we anticipated, the set of equations (1)-(5) is actually inconsistent with the separation of variables assumed in (7)-(10). That is, the only solution that satisfies such separation of variables is the trivial solution with $n, u_{z}, u_{r}, u_{\theta}$ all constant. However, the separation of variables is still worth considering if one can derive a class of approximate solutions for our model that are much faster to compute than the exact numerical solution, and its error relative to the full solution is small.
It is straightforward to prove that equation (4) is automatically satisfied by using the factorization assumption in equations (7)-(10). Substituting equations (7)-(9) into equations (1)-(5) and separating each equation in $\eta$ and $z$ dependent terms, one can get the following set of equations for $u_{c}(z), n_{c}(z)$ and $a(z)$ :

$$
\begin{gathered}
a^{2} n_{c} u_{c}=A, \\
\frac{1}{2} u_{c}^{2}+\frac{\gamma}{\gamma-1} n_{c}^{\gamma-1}=B, \\
n_{c}=a^{D}, \\
\frac{u_{c} a}{n_{c}^{\gamma-1}}\left(a^{\prime} u_{c}\right)^{\prime}-\frac{E^{2}}{a^{2} n_{c}^{\gamma-1}}=\gamma C,
\end{gathered}
$$

and the following equations for $n_{t}(\eta)$ and $u_{t}(\eta)$ :

$$
\begin{aligned}
n_{t}^{2-D(\gamma-1)} & =u_{t}^{-2 D}, \\
\frac{n_{t}^{\gamma-2} n_{t}^{\prime}}{\eta u_{t}^{2}} & =-C,
\end{aligned}
$$

where $A, B, C$ and $D$ are arbitrary (separation) constants. Details of the calculation can be found in the appendix. Note that equation (12) descends from the continuity equation (1), 


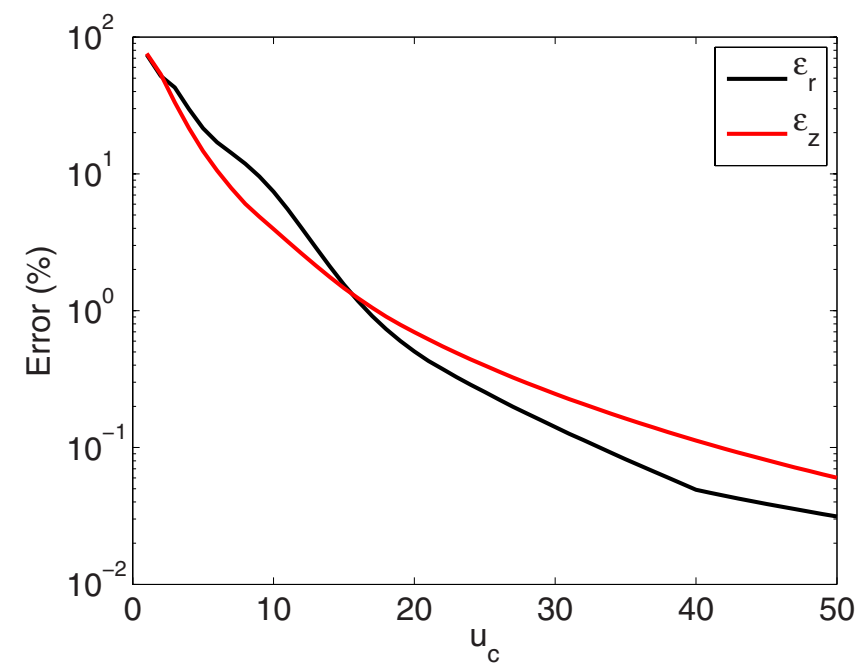

Figure 3. PK solution. Errors $\varepsilon_{r}$ (black) and $\varepsilon_{z}$ (red) as function of $u_{c}$.

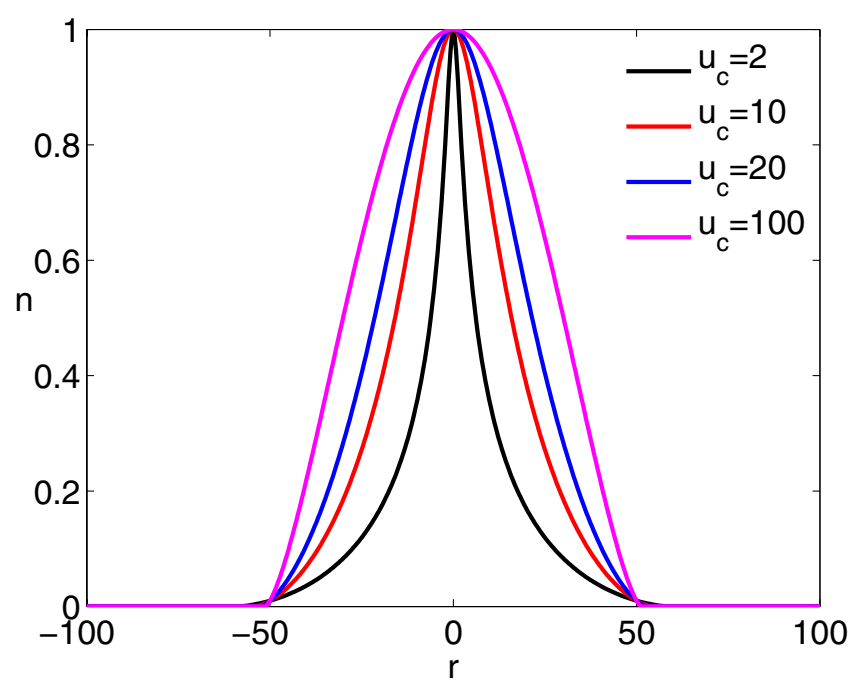

Figure 4. AF solution. Profile of the density $n$ at the injection boundary $z=0$, for $u_{c}=2,10,20,100$ (in black, red, blue and magenta, respectively). In the region $r \gtrsim 50$ where the density becomes imaginary, it is artificially set equal to zero.

equations (13), (14), (16) from the momentum equation in the $z$ direction (3), and equations (15) and (17) from the momentum equation in the $r$ direction (2). Clearly, we have a set of six equations for only five unknowns, and the system is overdetermined.

\section{Approximate solutions for $u_{\theta}=0$}

In order to derive an approximate solution of the model, a common approach is to assume $u_{c}=$ const and to prescribe a certain profile for $u_{t}$. In this way one can still satisfy some (but not all) of the equations (12)-(17). For simplicity, and to be adherent to earlier literature, we consider in this section the case where $E=0$, and thus $u_{\theta}=0$. All of the solutions discussed here satisfy the continuity equation and the conservation of momentum in $r$, but not in $z$. From equations

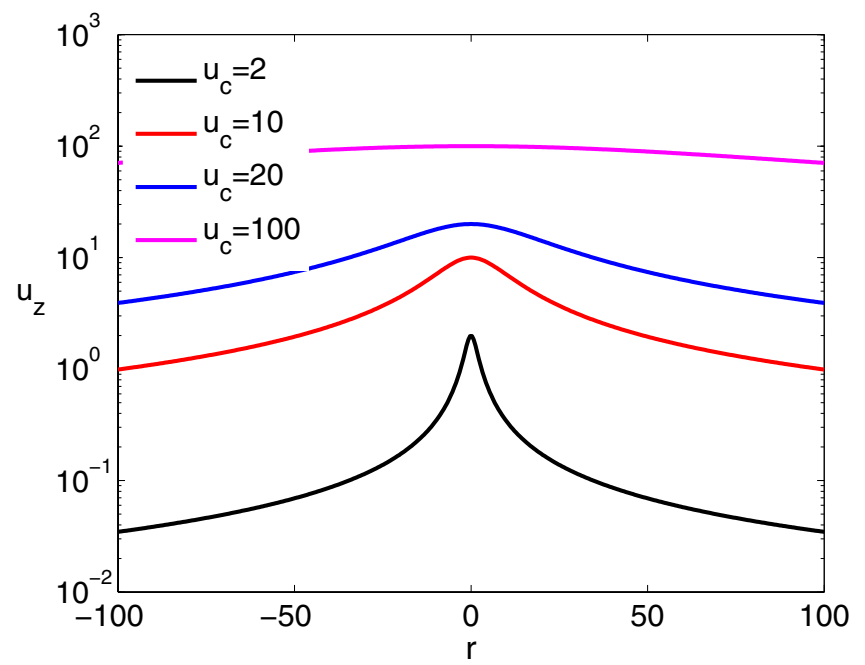

Figure 5. AF solution. Profile of the axial velocity $u_{z}$ at the injection boundary $z=0$, for $u_{c}=2,10,20,100$ (in black, red, blue and magenta, respectively). Vertical axis in logarithmic scale.

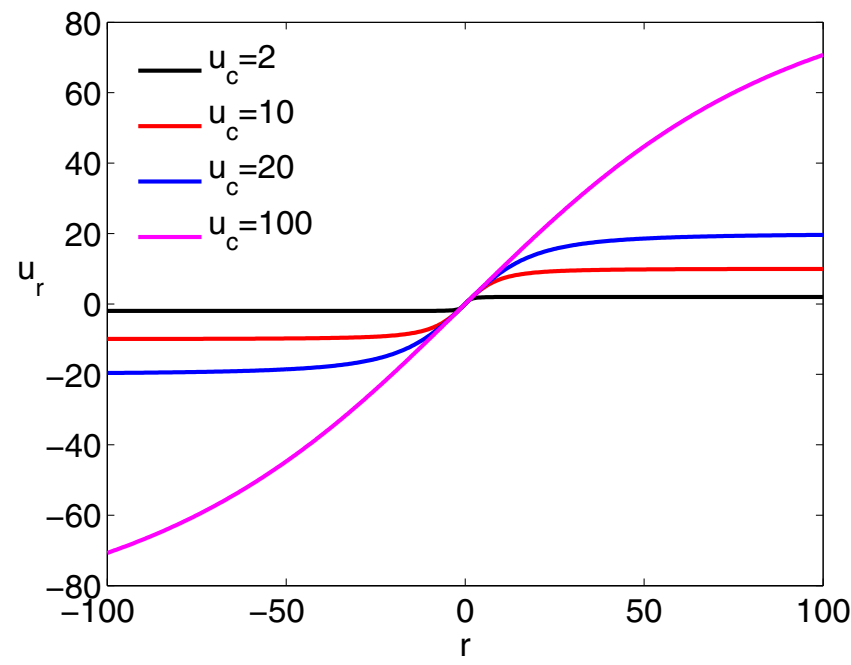

Figure 6. AF solution. Profile of the radial velocity $u_{r}$ at the injection boundary $z=0$, for $u_{c}=2,10,20,100$ (in black, red, blue and magenta, respectively).

(12), (15) and (17), it follows that

$$
\begin{gathered}
n_{c}(z)=\frac{a^{2}(0) n_{c}(0)}{a^{2}(z)}, \\
a^{\prime \prime}(z)=a^{1-2 \gamma}(z) \frac{\gamma C}{u_{c}^{2}} a(0)^{2(\gamma-1)} n_{c}(0)^{1-2 \gamma}, \\
n_{t}^{\gamma-2} n_{t}^{\prime}=-\eta u_{t}^{2} C .
\end{gathered}
$$

In addition to the value of $u_{c}$ and the profile of $u_{t}$, equations (18)-(20) require to specify the value of $C, a(0), a^{\prime}(0)$ and $n_{c}(0)$. As we will see some of these free parameters depend on the boundary conditions at $z=0$, i.e. the boundary from which the plume is injected. We assume the following boundary conditions:

$$
\begin{gathered}
n(r=0, z=0)=1, \\
n(r=R, z=0)=0.01,
\end{gathered}
$$



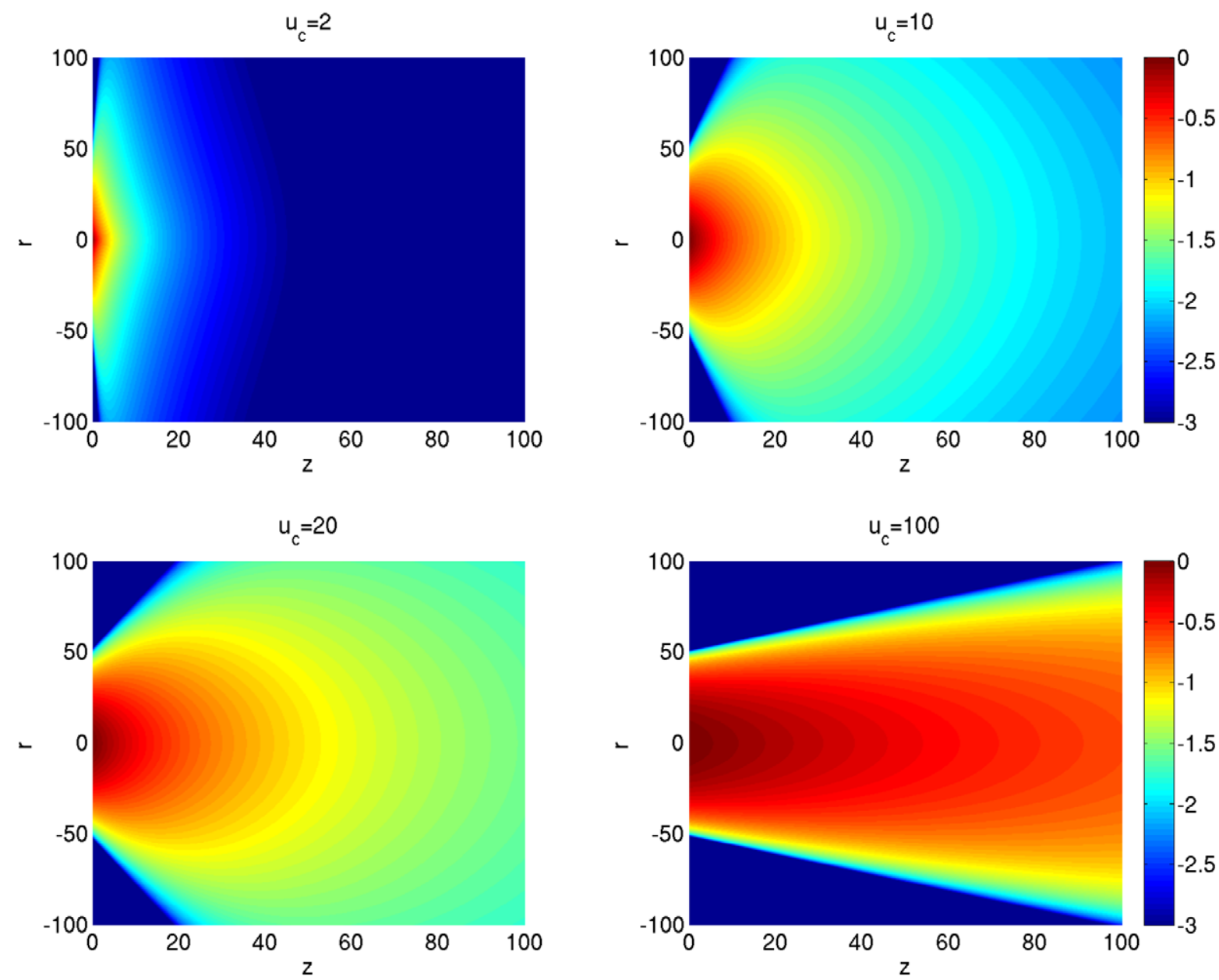

Figure 7. AF solution. Two-dimensional profiles of the density in $(z, r)$, for $u_{c}=2,10,20,100$, in logarithmic scale. The density is artificially set equal to zero in the regions where it becomes imaginary.

$$
\begin{gathered}
u_{r}(r=1, z=0)=1, \\
\left.\frac{\partial n}{\partial r}\right|_{r=0}=0, \\
\left.\frac{\partial u_{z}}{\partial r}\right|_{r=0}=0, \\
u_{r}(r=0, z)=0 .
\end{gathered}
$$

Equations (21) and (23) are simply normalization constraints. Equation (22) requires that the profile of the density at injection decreases by a factor of 100 within the characteristic length $R$. Equations (24)-(26) are symmetry conditions at $r=0$.

In this section, we describe the solutions presented in [1-3], and we propose a new class of solutions that includes earlier models as limiting cases and produces a higher degree of flexibility in terms of plume injection profiles that can be represented at $z=0$.

Such approximate solutions provide the density and velocity profiles at the boundary $z=0$ which are used to calculate the (exact) numerical solution. The latter is evaluated on a domain $[r, z]=[0, R] \times[0,80]$, with $R=50$ and grid size $\Delta r=\Delta z=0.2$. The derivatives in $r$ and $z$ are discretized with a fourth order central (5-points stencil) and a third order upwind (4-points stencil) difference scheme, respectively.
Since we are only interested in the steady-state solution, we have used the following marching scheme for solving numerically equations (1)-(5). First, the solution is calculated on the partial domain $[0, R] \times[0,4 \Delta z]$ with an iterative Newton-GMRES solver [26]. The solution is then extended to the full domain by adding one row of cells in $z$ at the time and calculating the new solution at each step. This is possible since we use an upwind discretization in $z$ that requires only the points where the solution has already been calculated.

For each approximate solution we evaluate the error with respect to the full numerical solution. We define the following two measures of error:

$$
\begin{aligned}
& \varepsilon_{r}=\max \left|\frac{\left(n^{a} u_{r}^{a}-n^{n} u_{r}^{n}\right)}{n^{n} u_{r}^{n}}\right| \cdot 100, \\
& \varepsilon_{z}=\max \left|\frac{\left(n^{a} u_{z}^{a}-n^{n} u_{z}^{n}\right)}{n^{n} u_{z}^{n}}\right| \cdot 100,
\end{aligned}
$$

which are the percentage $L_{1}$ norm of the relative error of the radial and axial fluxes, where the superscripts $a$ and $n$ indicate the approximate and the numerical solutions, respectively. Each of the solutions presented in [1-3] makes a different assumption on $u_{t}$, and we proceed to discuss them separately. 


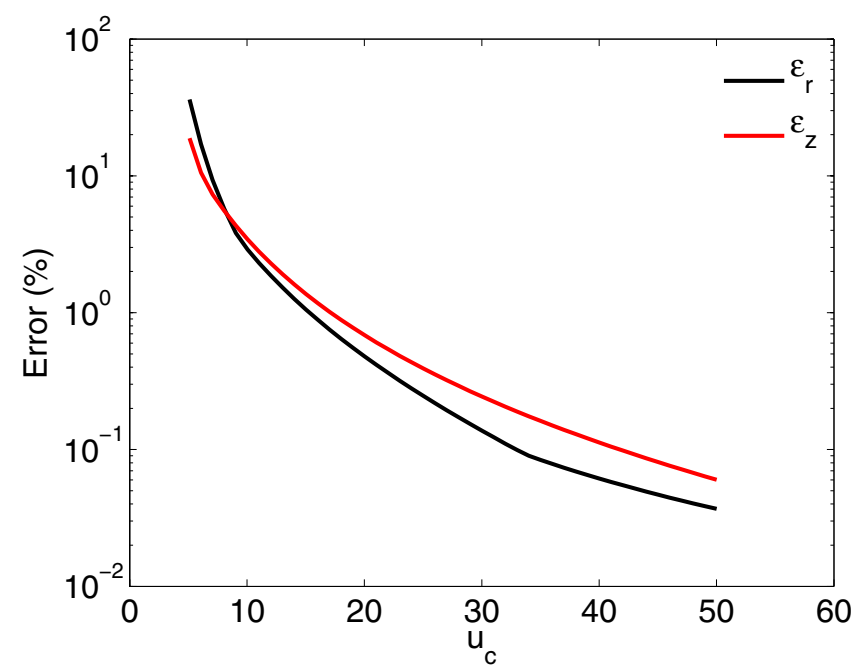

Figure 8. AF solution. Errors $\varepsilon_{r}$ (black) and $\varepsilon_{z}$ (red) as function of $u_{c}$.

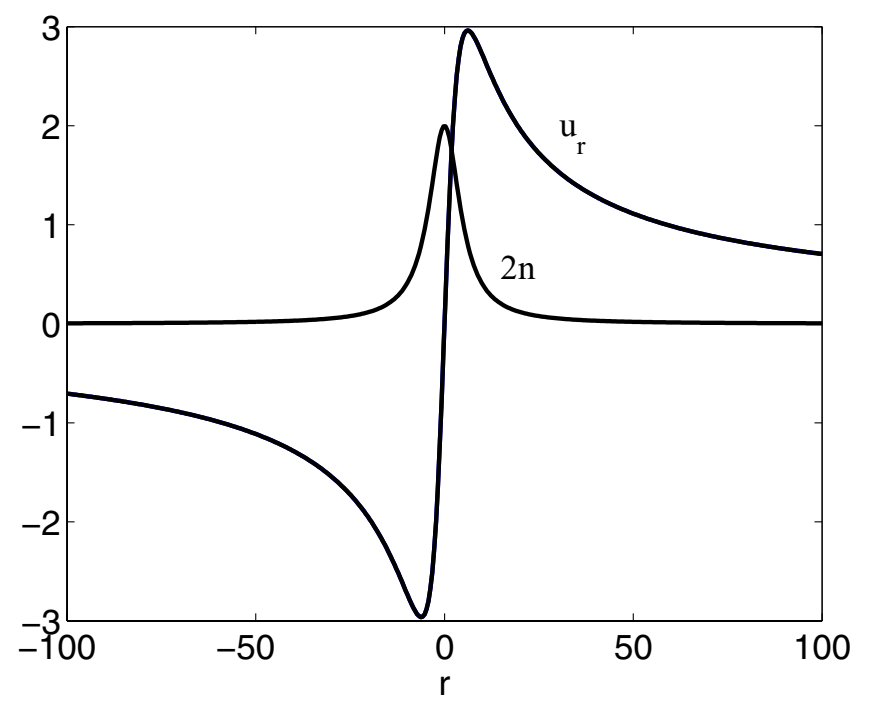

Figure 9. KT solution. Profiles for the density $n$ (rescaled by a factor of 2) and the radial velocity $u_{r}$ at the injection boundary $z=0$.

\subsection{Parks and Katz (PK) solution}

The solution proposed in [1] assumes a constant $u_{t}=1$. Solving equation (20) for $n_{t}$ gives the profile:

$$
n_{t}=\left(C_{0}-\frac{\gamma-1}{2} C \eta^{2}\right)^{\frac{1}{\gamma-1}}
$$

where $C_{0}$ is an integration constant. Following [25] we set $C_{0}=1$, therefore the PK solution reads:

$$
\begin{gathered}
n(r, z)=\frac{a^{2}(0)}{a^{2}(z)}\left(1-\frac{\gamma-1}{2} C \frac{r^{2}}{a^{2}(z)}\right)^{\frac{1}{\gamma-1}}, \\
u_{r}(r, z)=\frac{r}{a(z)} a^{\prime}(z) u_{c} \\
u_{z}(r, z)=u_{c}
\end{gathered}
$$

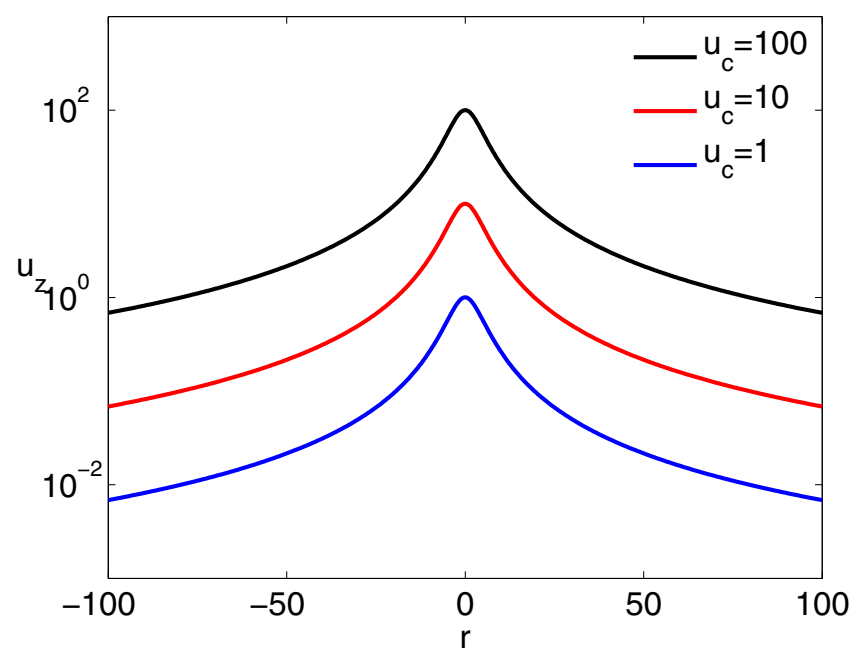

Figure 10. KT solution. Profile for the axial velocity $u_{z}$ at the injection boundary $z=0$, for $u_{c}=1,10,100$ (in blue, red, and black, respectively). The vertical axis is in logarithmic scale.

where we have used $n_{c}(0)=1$, imposed by equation (21). The boundary condition (22) allows to solve for $C$ and equation (23) yields the relationship

$$
a(0)=u_{c} a^{\prime}(0) .
$$

In summary, the only two free parameters are $u_{c}$ and $a^{\prime}(0)$ and the profile for $a(z)$ can be calculated by solving numerically equation (19). Note that the profiles of $u_{r}$ and $n$ at the injection boundary $z=0$ do not depend on the particular choice of $u_{c}$ and $a^{\prime}(0)$. Figure 1 shows the profiles for the density and the radial velocity at $z=0$ as a function of $r$. Clearly, the radial velocity increases linearly with $r$. An important feature of the PK solution is that, for any given value of $z$, the solution is constrained to a certain range in $r$. Indeed, one can see from equation (30) that the density becomes a complex quantity (i.e. unphysical) for a large enough $r$. In figure 1 we have artificially set $n=0$ in the regions where the density becomes imaginary. Figure 2 shows the density profile in the $(z, r)$ plane, for different values of the axial velocity $u_{z}=u_{c}$, in logarithmic scale, for a fixed value of $a^{\prime}(0)=0.2$. Once again, notice that the density profile at $z=0$ is not a function of $u_{c}$. The errors $\varepsilon_{r}$ and $\varepsilon_{z}$ as a function of $u_{c}$ are shown in figure 3 . For $u_{c}>20, \varepsilon_{r}$ and $\varepsilon_{z}$ are smaller than $1 \%$. Note, however, that here and in the next similar figures, the error has been calculated in the restricted region $|r| \leqslant R$. The region where the density is artificially set equal to zero is excluded by the evaluation of the error. The reason is that the marching routine described above is not very robust when a sharp gradient in the density is included as boundary condition and, therefore, it is not straightforward to obtain a numerical solution that extends to $|r|>R$.

\subsection{Ashkenazy and Fruchtman (AF) solution}

The AF solution [3] assumes a conical velocity profile at the injection, such that

$$
u_{t}=\left(1+a^{\prime}(0)^{2} \eta^{2}\right)^{-\frac{1}{2}} .
$$



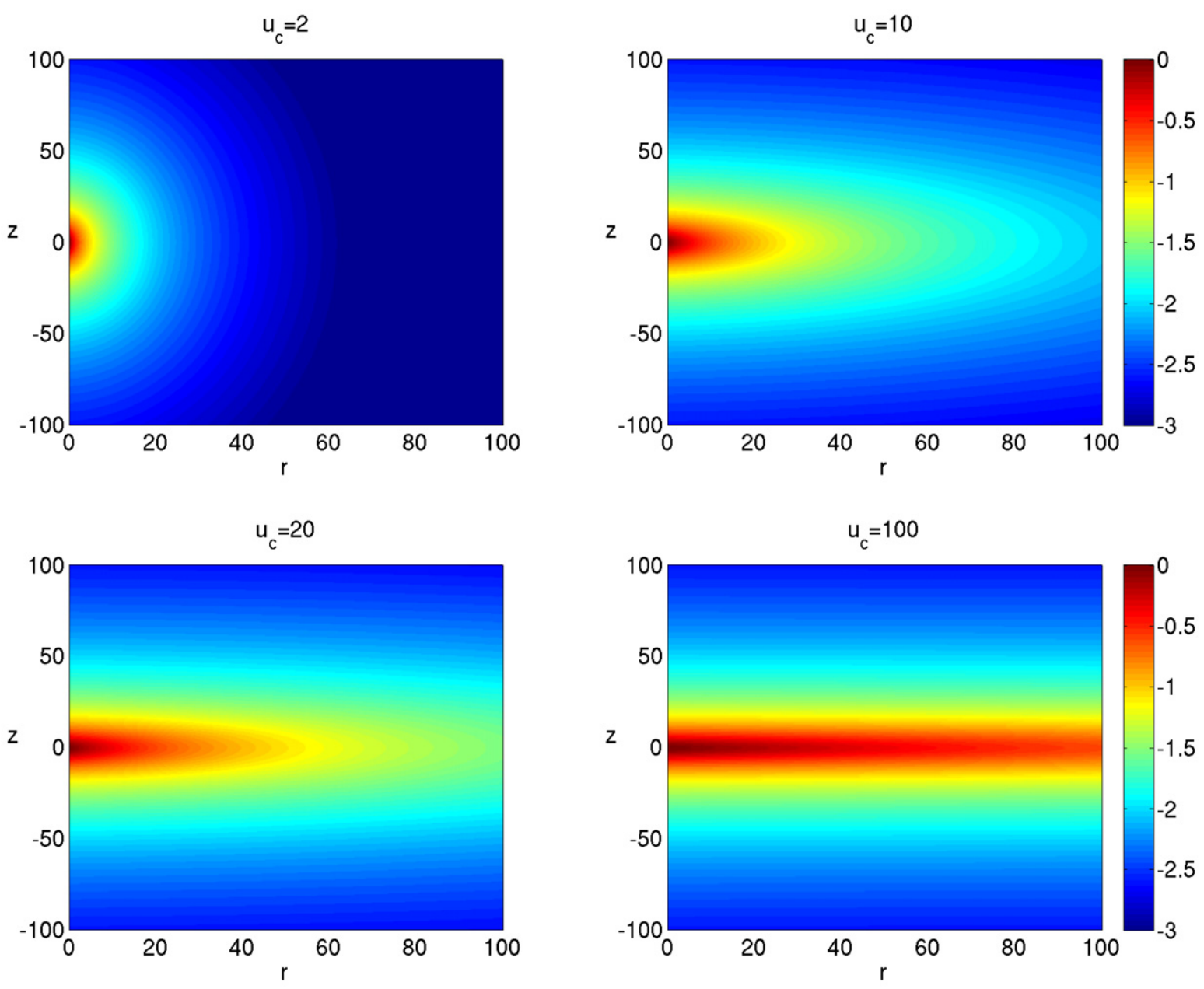

Figure 11. KT solution. Two-dimensional profiles of the density in $(z, r)$, for $u_{c}=2,10,20,100$, in logarithmic scale.

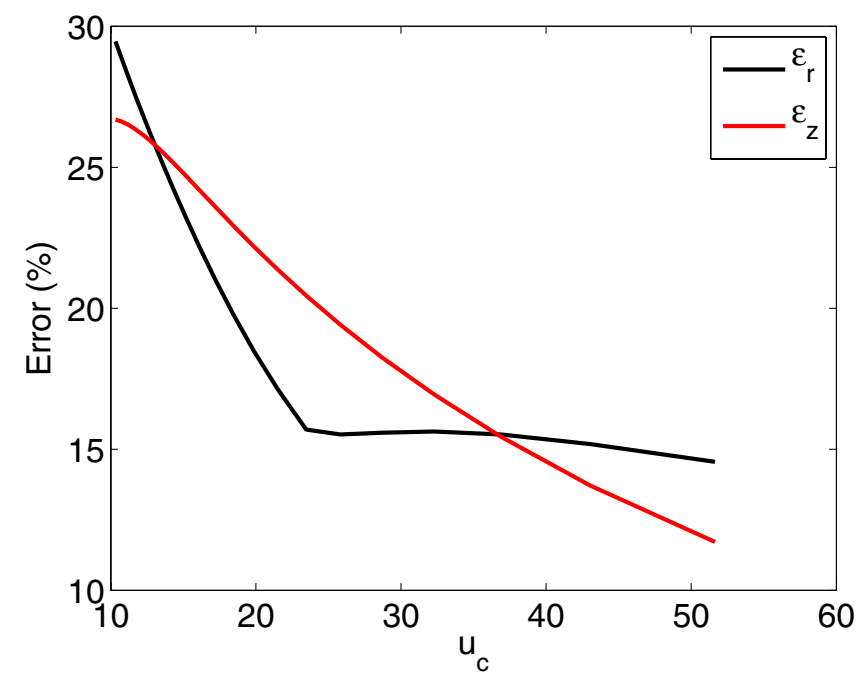

Figure 12. PK solution. Errors $\varepsilon_{r}$ (black) and $\varepsilon_{z}$ (red) as function of $u_{c}$.

The profile for $n_{t}$ is given by solving equation (20):

$$
n_{t}=\left(C_{0}-\frac{C(\gamma-1)}{2\left(a^{\prime}(0)\right)^{2}} \log \left(1+\left(a^{\prime}(0)\right)^{2} \eta^{2}\right)\right)^{\frac{1}{\gamma-1}}
$$

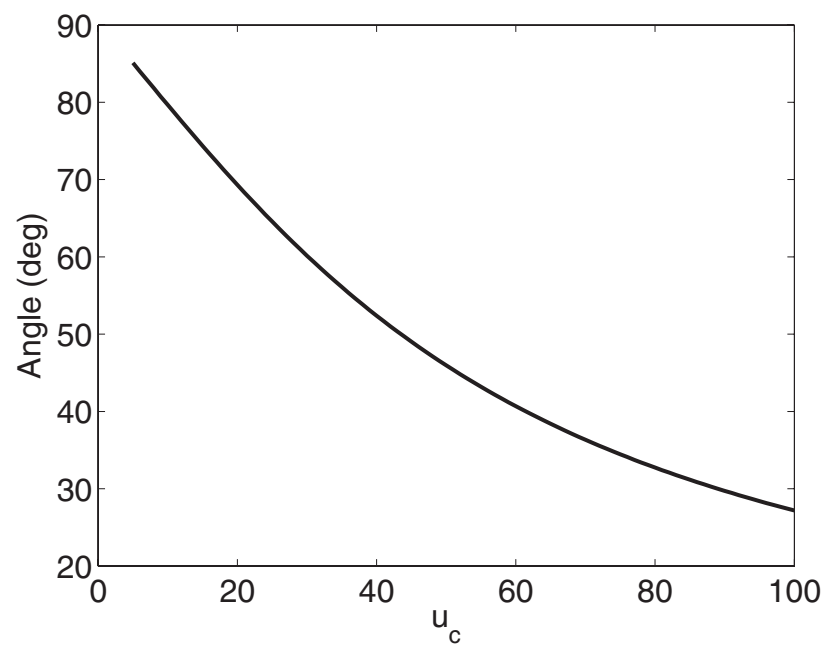

Figure 13. $\mathrm{PK}$ and $\mathrm{AF}$ solutions. Angle (in degrees), with respect to the axis $r=0$, that determines the (approximately straight) isocontour $n=0$, as a function of the injection velocity $u_{c}$. The expansion is bounded within this cone angle.

where $C_{0}$ is an integration constant that we set equal to 1 . The boundary condition (21) imposes $n_{c}(0)=1$, while equation (22) yields an equation that must be solved to obtain the value of $C$. The remaining free parameters $u_{c}, a(0), a^{\prime}(0)$ 


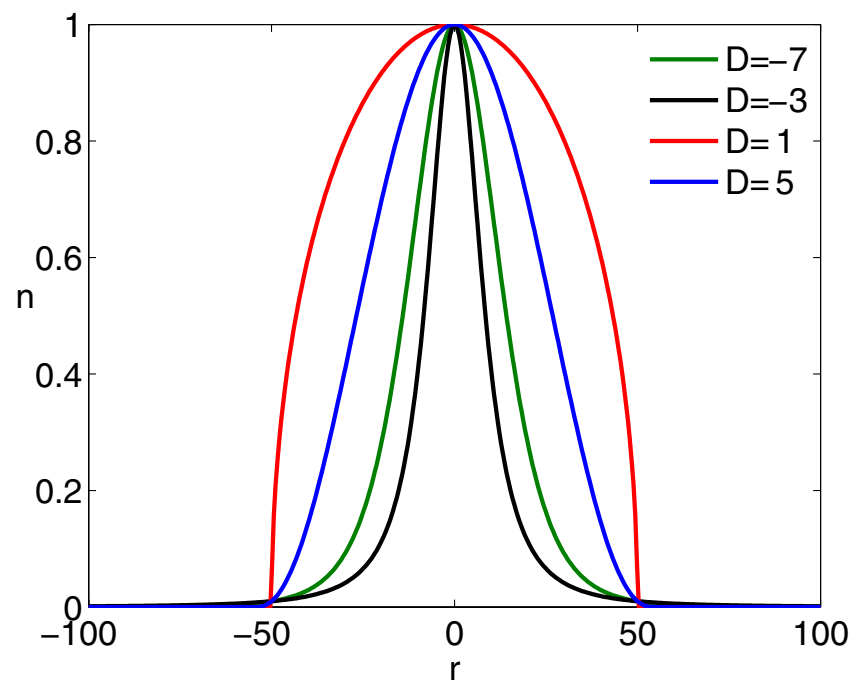

Figure 14. Generalized solution. Density profile at the injection boundary $z=0$ for $D=-7,-3,1,5$ (in green, black, red and blue, respectively).

obey, via the boundary condition (23), the relationship

$$
\frac{a(0)}{a^{\prime}(0)} \sqrt{1+\left(\frac{a^{\prime}(0)}{a(0)}\right)^{2}}=u_{c} .
$$

For simplicity, we fix $a^{\prime}(0)=0.2$, allowing $u_{c}$ to vary, and calculate $a(0)$ through equation (36). As usual, $a(z)$ is calculated via equation (19). In summary, the profiles for $n$, $u_{r}$ and $u_{z}$ are

$$
\begin{aligned}
& n(r, z)=\left(\frac{a(0)}{a(z)}\right)^{2} \\
& \times\left[1-\frac{C(\gamma-1)}{2\left(a^{\prime}(0)\right)^{2}} \log \left(1+\left(\frac{a^{\prime}(0)}{a(z)} r\right)^{2}\right)\right]^{\frac{1}{\gamma-1}}, \\
& u_{r}(r, z)=\frac{r}{a(z)} a^{\prime}(z) u_{c} \\
& \times\left[1-\frac{C(\gamma-1)}{2\left(a^{\prime}(0)\right)^{2}} \log \left(1+\left(\frac{a^{\prime}(0)}{a(z)} r\right)^{2}\right)\right]^{\frac{1}{\gamma-1}}, \\
& u_{z}(r, z)=\frac{u_{c}}{\sqrt{1+\left(\frac{a^{\prime}(0)}{a(z)} r\right)^{2}}} .
\end{aligned}
$$

Figure 4 shows the density profile for the AF solution at the injection boundary $z=0$, as function of $r$, for the values $u_{c}=2,10,20,100$. Similarly to the previous model, the density becomes imaginary for $r$ larger than certain values. The corresponding profiles for $u_{z}$ and $u_{r}$ are plotted in figures 5 and 6 , respectively. Figure 7 shows the two-dimensional density profile in the $(z, r)$ plane, in logarithmic scale for four values of $u_{c}$. One can notice a qualitative similarity with the profiles of the PK solution, especially for large $u_{c}$. The errors for the AF solution are shown in figure 8 and are comparable with those obtained for the PK solution (figure 3), i.e. the errors are smaller than $1 \%$, for $u_{c} \gtrsim 20$.

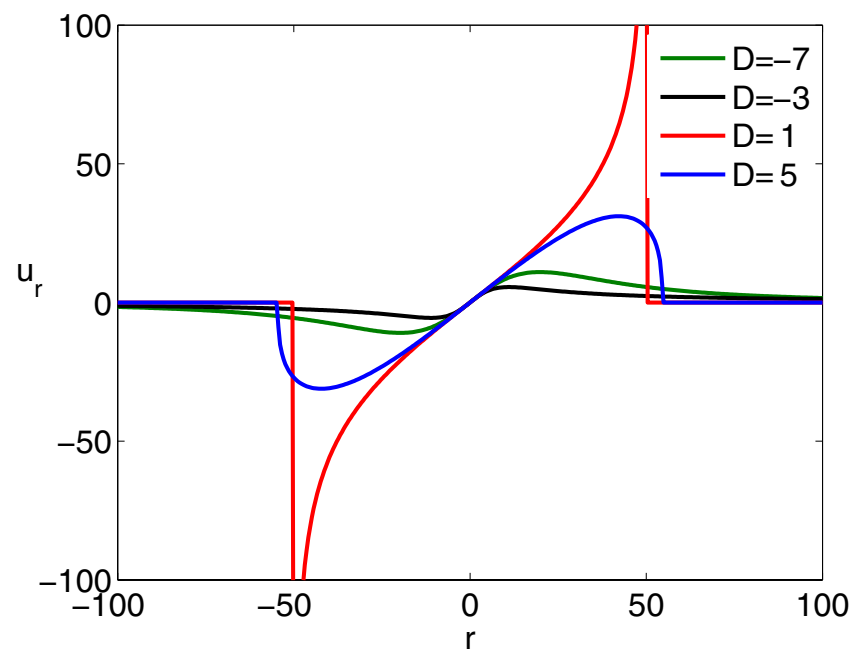

Figure 15. Generalized solution. Radial velocity profile at the injection boundary $z=0$ for $D=-7,-3,1,5$ (in green, black, red and blue, respectively).

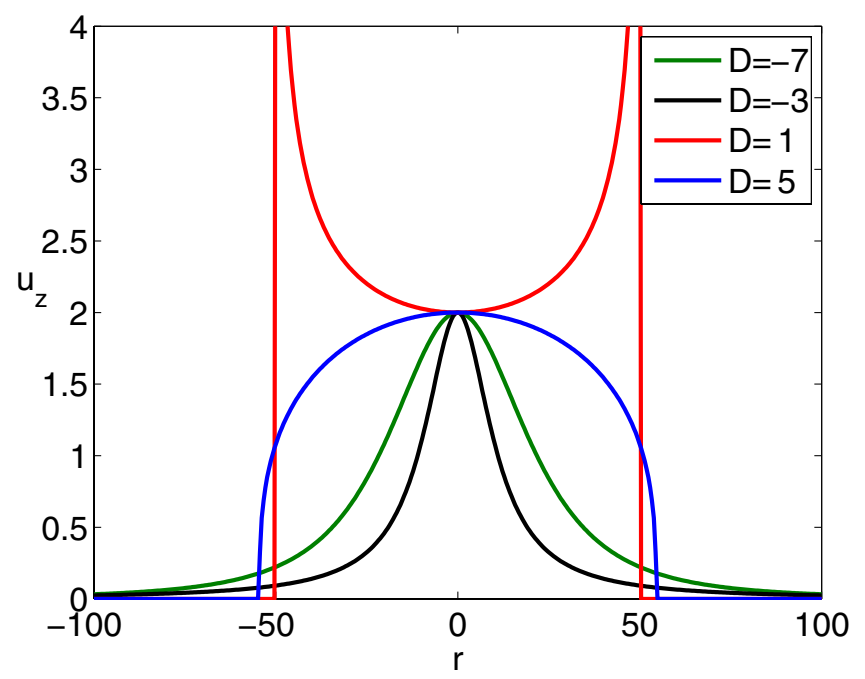

Figure 16. Generalized solution. Axial velocity profile at the injection boundary $z=0$ for $D=-7,-3,1,5$ (in green, black, red and blue, respectively).

\subsection{Korsun and Tverdokhlebova (KT) solution}

The solution proposed in [2] assumes the profile

$$
u_{t}(\eta)=\left(1-\frac{C}{2} \eta^{2}\right)^{-\frac{\gamma}{2}}
$$

from which equation (20) dictates

$$
n_{t}(\eta)=\left(1-\frac{C}{2} \eta^{2}\right)^{-1}
$$

Moreover, by setting $D=-2$, the KT solution satisfies equation (16). By applying the boundary condition (21) and equation (14), it follows that $a(0)=n_{c}(0)=1$. In summary, the KT solution not only satisfies the equations of continuity and momentum in $r$, but also equations (14) and (16), i.e. it satisfies two out of three equations for the conservation of 

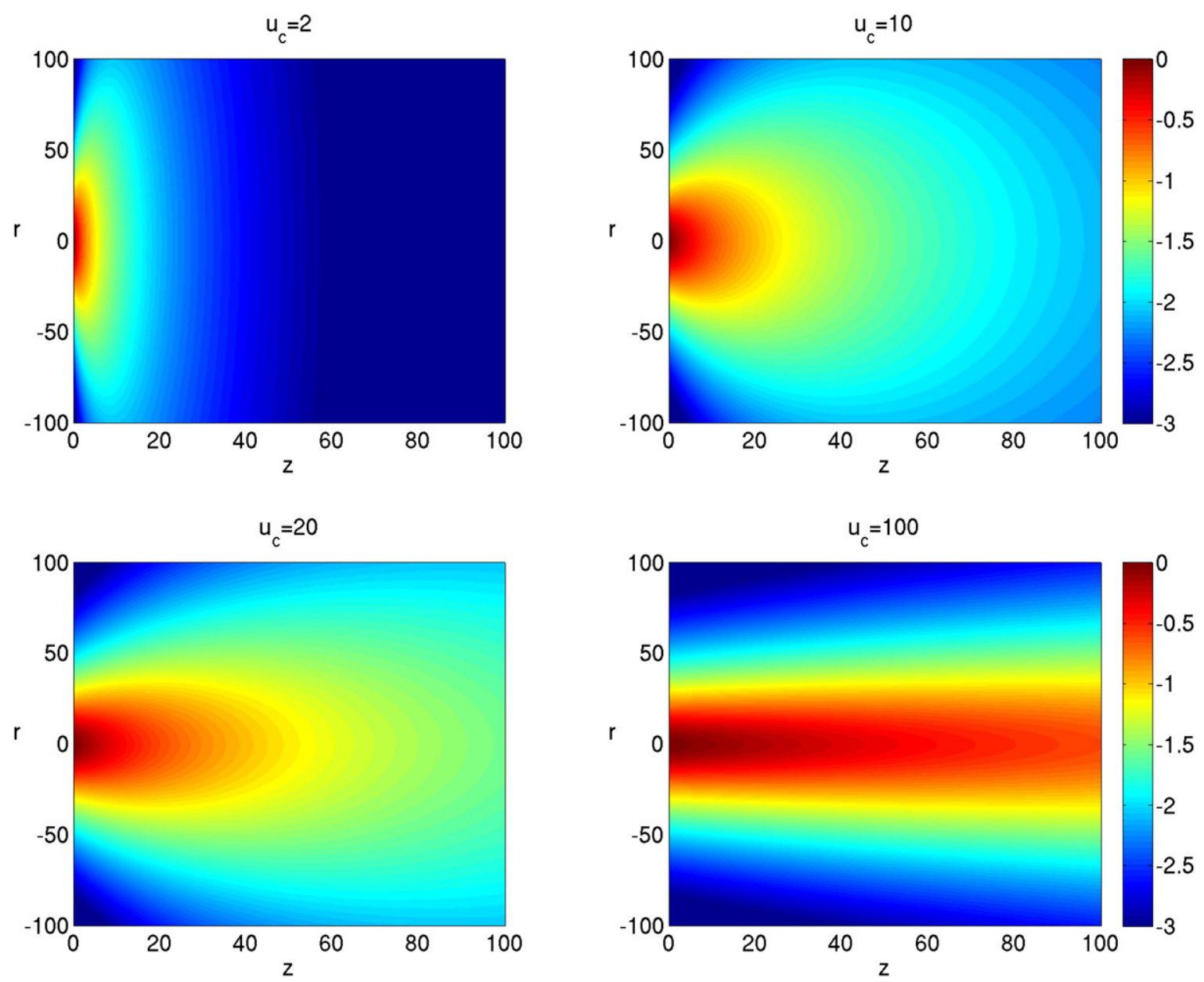

Figure 17. Generalized solution. Two-dimensional profiles of the density in $(z, r)$, for $u_{c}=2,10,20,100$, and $D=-5$, in logarithmic scale.

momentum in the $z$ direction. The profiles for $n, u_{r}$ and $u_{z}$ are

$$
\begin{gathered}
n(r, z)=\frac{1}{a^{2}(z)}\left(1-\frac{C}{2} \frac{r^{2}}{a^{2}(z)}\right)^{-1}, \\
u_{r}(r, z)=\frac{r}{a(z)} a^{\prime}(z) u_{c}\left(1-\frac{C}{2} \frac{r^{2}}{a^{2}(z)}\right)^{-\frac{\gamma}{2}}, \\
u_{z}(r, z)=u_{c}\left(1-\frac{C}{2} \frac{r^{2}}{a^{2}(z)}\right)^{-\frac{\gamma}{2}} .
\end{gathered}
$$

The boundary condition (22) provides the expression for $C=2\left(1-\frac{1}{0.01}\right) / R^{2}=-0.0792$, and $a(z)$ is calculated via equation (19). Hence, the only free parameter is $u_{c}$ which is related to $a^{\prime}(0)$ through the relationship

$$
a^{\prime}(0)=\frac{1}{u_{c}}\left(1-\frac{C}{2}\right)^{\frac{\gamma}{2}},
$$

that is obtained by applying equation (23).

Similarly to the PK solution, the density and radial velocity profiles at the injection boundary $z=0$ are not a function of $u_{c}$. They are shown in figure 9. The profile for $u_{z}$ is a linear function of $u_{c}$. Three examples for the values $u_{c}=1,10,100$ are shown in figure 10. Four examples of two-dimensional density profiles are shown in logarithmic scale in figure 11 , for $u_{c}=2,10,20,100$. The errors $\varepsilon_{r}$ and $\varepsilon_{z}$ as a function of $u_{c}$ are shown in figure 12. Notice that they are much higher than the respective errors for the PK and AF solutions. This might be surprising since the KT solution satisfies equations (14) and (16), while the PK and AF solutions do not. However, given the nonlinear nature of the model, one cannot predict how large the errors will be with respect to the true solution. Moreover, as discussed in the appendix, equations (14) and (16) follow from the assumption that equation (13) holds. Hence, satisfying equations (14) and (16) does not automatically yield a lower error, since equation (13) is still not satisfied.

\subsection{Discussion on the PK, AF and KT solutions}

We have noted a certain similarity between the PK and AF solutions for large $u_{c}$. This is not surprising if one realizes that the AF solution tends exactly to the PK solution in the limit $u_{c} \rightarrow \infty$. Indeed, in this limit, equation (36) requires that $a(0) \rightarrow \infty$ and/or $a^{\prime}(0) \rightarrow 0$. Taylor expanding the logarithmic term in $n$ and $u_{r}$ (equations (37) and (38)) one has

$$
\log \left(1+\left(\frac{a^{\prime}(0)}{a(z)} r\right)^{2}\right) \rightarrow\left(\frac{a^{\prime}(0) r}{a(z)}\right)^{2}
$$

and equations (37)-(39) reduce exactly to equations (30)-(32). 

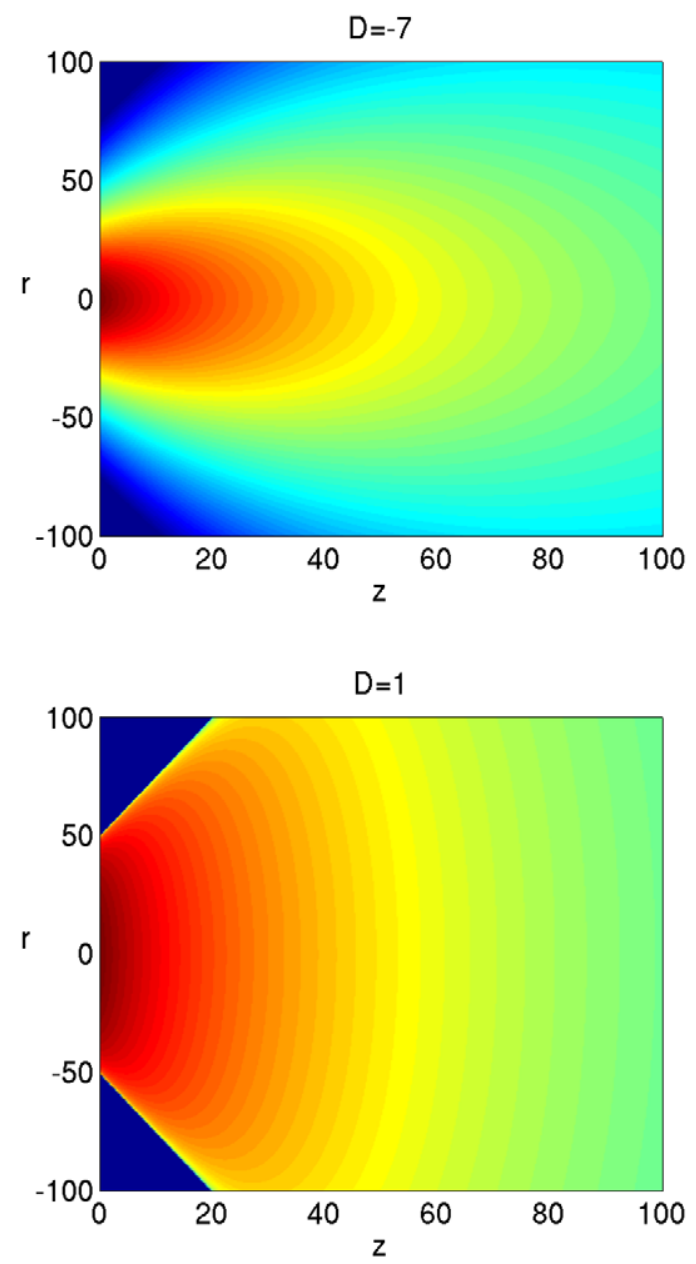
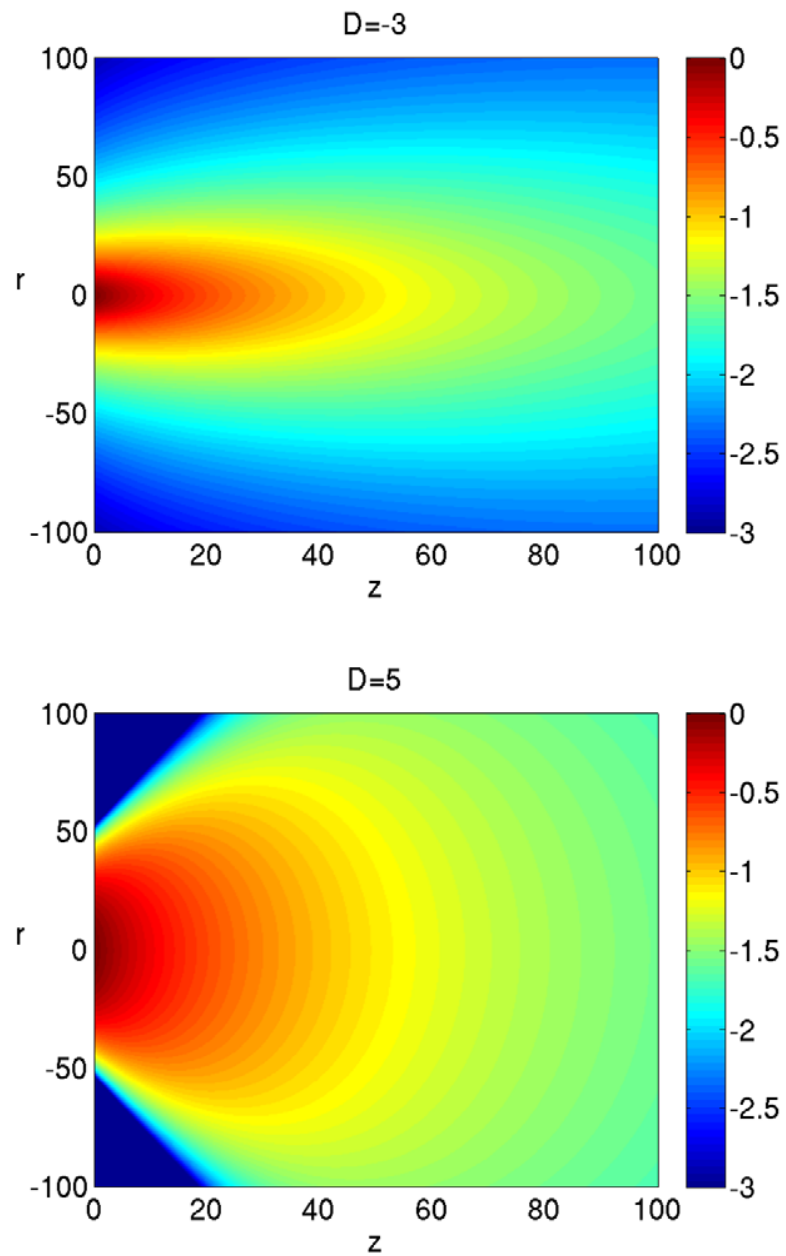

Figure 18. Generalized solution. Two-dimensional profiles of the density in $(z, r)$, for $D=-7,-3,1,5$, and $u_{c}=20$, in logarithmic scale.

Although the PK and AF solutions yield relatively good results in terms of small errors with respect to the numerical solutions (especially for large $u_{c}$ ), they are severely limited in the choice of density and velocity profiles at the injection. In fact, the value of $u_{c}$ determines, by construction, the injection profiles that, in turn, determine the region in which the plume propagates. From figures 2 and 7, it is evident that the cone of propagation becomes more and more collimated as $u_{c}$ increases. In figure 13, we show the angle (in degrees) of the isocontour $n=0$ with respect to the axis $r=0$, as a function of $u_{c}$. Note that the cone of propagation is identical for the AF and PK solutions. Also, although the isocontour $n=0$ is not exactly a straight line in the $(z, r)$ plane, it can be considered approximately straight in the region plotted in figures 2 and 7. We emphasize that this cone of propagation, and the corresponding angles shown in figure 13 are an intrinsic characteristic of the PK and AF solutions, which depends solely on the value of $u_{c}$. On the other hand, the user of such approximate solutions might need to be able to choose a different injection profile than the one imposed by these solutions, as this will depend on the source generating the contactor plasma. In particular, as highlighted in figure 13, large injection angles are not possible for a large injection velocity, for the $\mathrm{PK}$ and $\mathrm{AF}$ solutions. Differently from PK and AF, the KT solution does not involve a propagation cone. Indeed, from equation (42), it is easy to see that the density tends to zero only asymptotically for $r \rightarrow \infty$. Unfortunately, although the KT profiles offer an alternative to the PK and AF solutions, they yield a much larger error and therefore are not preferable.

In the following section we introduce a new class of solutions that allows a rather wide choice of injection profiles, still yielding relatively small errors with respect to the numerical solutions.

\section{A new class of solutions}

The new class of solutions is based on a generalization of the $\mathrm{KT}$ and PK solutions. Interestingly, their error is much smaller than the errors yield for the KT solution, and comparable to the ones of the PK and AF solutions. As in previous models, we still assume a constant $u_{c}$. We also assume the following profiles for $n_{t}$ and $u_{t}$ :

$$
\begin{gathered}
n_{t}(\eta)=\left(F-\frac{C}{D} \eta^{2}\right)^{\frac{D}{2}}, \\
u_{t}(\eta)=\left(F-\frac{C}{D} \eta^{2}\right)^{\frac{D(\gamma-1)}{4}-\frac{1}{2}} .
\end{gathered}
$$

Note that the KT solution belongs to this class of solution, for the particular case $D=-2$ and $F=1$, while for 


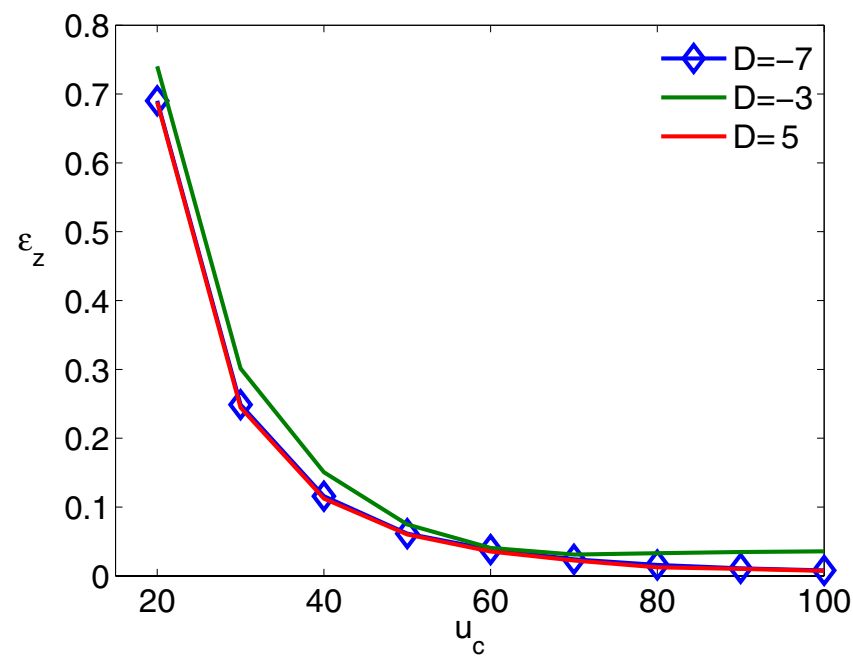

Figure 19. Generalized solution. Error $\varepsilon_{z}$ as a function of $u_{C}$ for $D=-7,-3,5$ (in blue, green and red, respectively), and $F=1$.

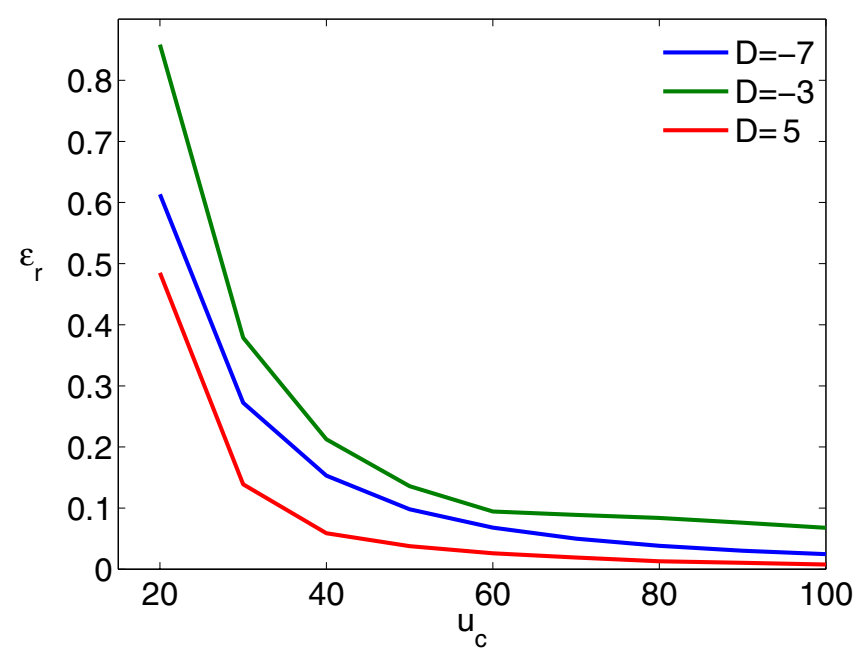

Figure 20. Generalized solution. Error $\varepsilon_{r}$ as a function of $u_{C}$ for $D=-7,-3,5$ (in blue, green and red, respectively), and $F=1$.

$D=2 /(\gamma-1)$ and $F=1$, one recovers the PK solution. Also, one can verify that equations (47)-(48) satisfy equation (16).

The solution reads:

$n(r, z)=\frac{a^{2}(0) n_{c}(0)}{a^{2}(z)}\left(F+\frac{C}{D} \frac{r^{2}}{a^{2}(z)}\right)^{\frac{D}{2}}$,

$u_{r}(r, z)=\frac{r}{a(z)} a^{\prime}(z) u_{c}\left(F+\frac{C}{D} \frac{r^{2}}{a^{2}(z)}\right)^{\frac{D(\gamma-1)}{4}-\frac{1}{2}}$,

$u_{z}(r, z)=u_{c}\left(F+\frac{C}{D} \frac{r^{2}}{a^{2}(z)}\right)^{\frac{D(\gamma-1)}{4}-\frac{1}{2}}$.

Applying the boundary conditions (21)-(23), one gets the following relationships:

$$
\begin{gathered}
n_{c}(0)=F^{-\frac{D}{2}}, \\
C=\frac{D a(0)^{2}}{R^{2}}(F(0.01-1))^{\frac{2}{D}}, \\
u_{c}=\frac{a(0)}{a^{\prime}(0)}\left(F+\frac{C}{D a(0)^{2}}\right)^{\frac{1}{2}-\frac{D(\gamma-1)}{4}} .
\end{gathered}
$$

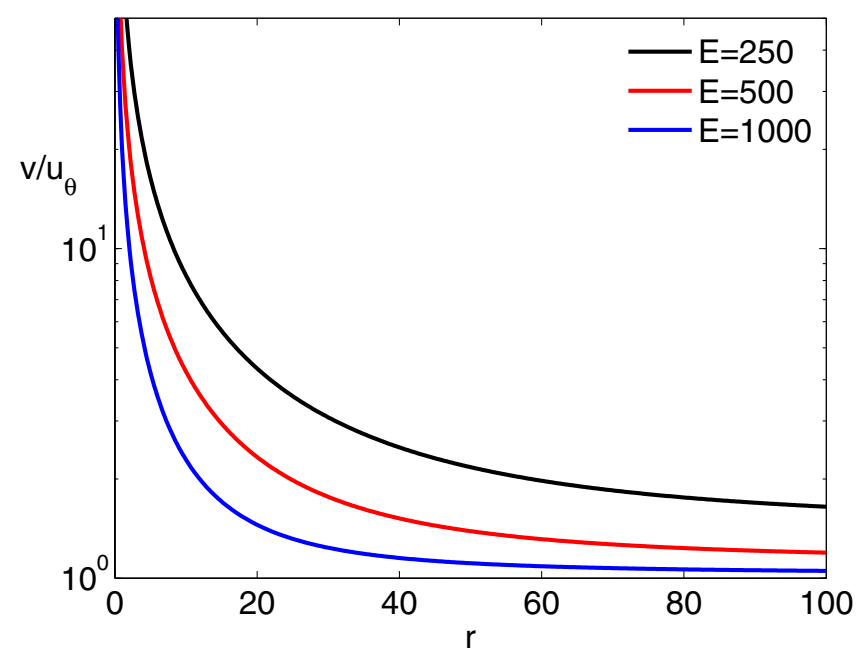

Figure 21. Ratio $v / u_{\theta}$ as a function of $r$ at $z=0$, for $E=250$ (black), 500 (red) and 1000 (blue).

It follows that four parameters can be freely chosen between $D, F, a(0), a^{\prime}(0)$ and $u_{c}$. In order to simplify the study and to be consistent with the previous models, we fix $a^{\prime}(0)=0.2$, $F=1$, and we vary $D$ and $u_{c}$ (solving equations (53)-(54) for $a(0)$ and $C)$. Note that the profiles of $n, u_{r}$ and $u_{z}$ at the injection boundary $z=0$ do not depend on $a^{\prime}(0)$.

Figure 14 shows the density profile at $z=0$ for $D=-7$ (green), -3 (black), 1 (red), 5 (blue), as a function of $r$. Clearly, the value of $D$ controls the width of the injected density, that can vary from Gaussian-like to a profile closer to a step function. Also, similar to the KT solution, the density tends to zero asymptotically when $D<0$, for $r \rightarrow \infty$. In this case, however, the value of $D$ determines the asymptotic scaling. On the other hand, for $D>0$, one has imaginary values that must be artificially set equal to zero. Figures 15 and 16 show the profiles of $u_{r}$ and $u_{z}$, respectively, for $D=-7$ (green), -3 (black), 1 (red), 5 (blue), obtained with $u_{c}=2$. Note that whilst the profiles of $n$ and $u_{r}$ do not change by varying $u_{c}$, the axial velocity $u_{z}$ is a linear function of $u_{c}$, i.e. the profiles in figure 16 are linearly rescaled by changing the value of $u_{c}$. In figure 17 we present a representative example of the two-dimensional density profile, in logarithmic scale, for $u_{c}=2,10,20,100$, and $D=-5$. In order to show the versatility of this class of solutions, we show more examples of the two-dimensional density profile in figure 18 , for $u_{c}=20$ and varying $D=-7,-3,1,5$. Clearly, the cases with $D<0$ do not present a propagation cone.

Figures 19 and 20 present the errors $\varepsilon_{z}$ and $\varepsilon_{r}$, as a function of $u_{c}$, for $D=-7$ (blue), -3 (green), 5 (red). As anticipated, the errors are comparable to the ones for the $\mathrm{PK}$ and AF solutions, and are monotonically decreasing with increasing $u_{c}$.

\section{Extension to the case $u_{\theta} \neq 0$}

By still considering a constant $u_{c}$, equation (19) is modified as

$$
\frac{u_{c}^{2} a a^{\prime \prime}}{n_{c}^{\gamma-1}}-\frac{E^{2}}{a^{2} n_{c}^{\gamma-1}}=\gamma C .
$$




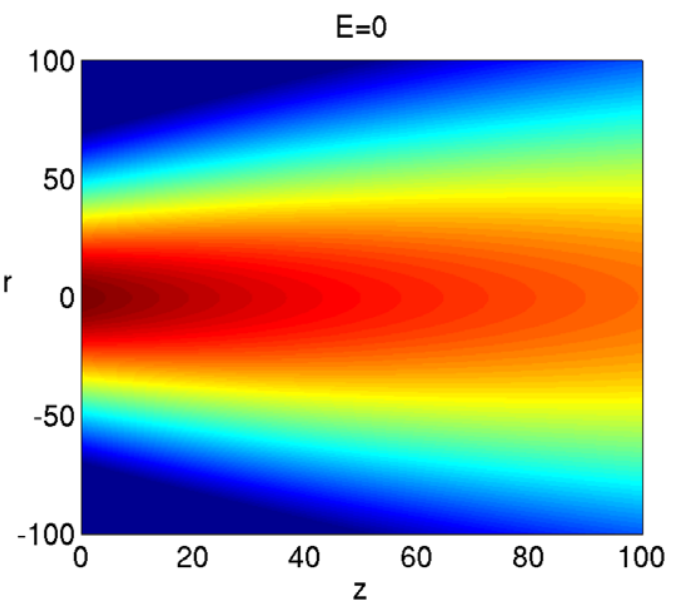

$E=1000$

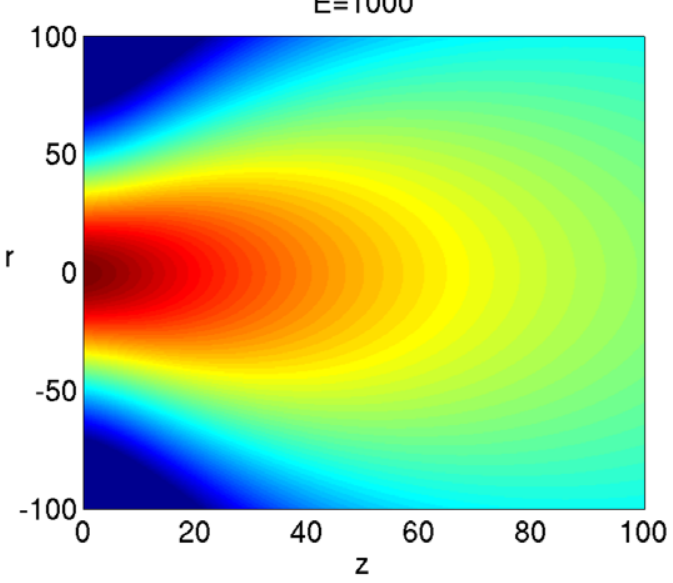

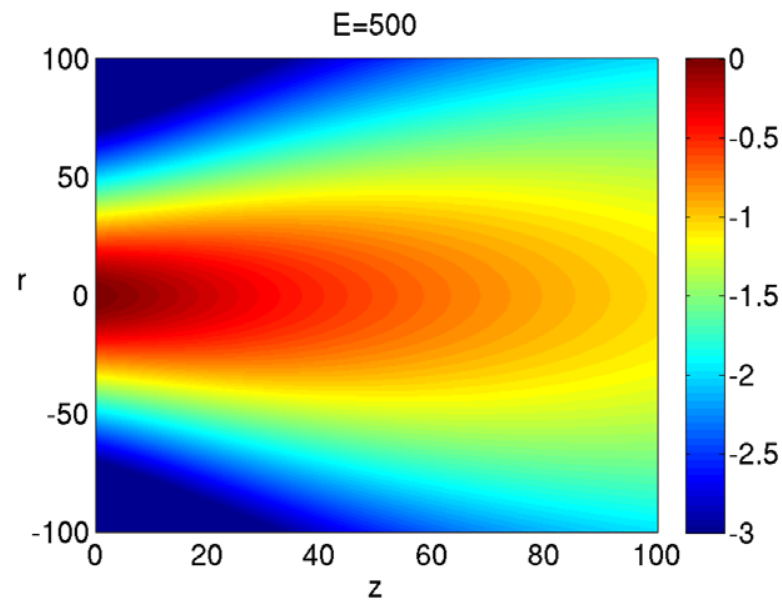

$E=2000$

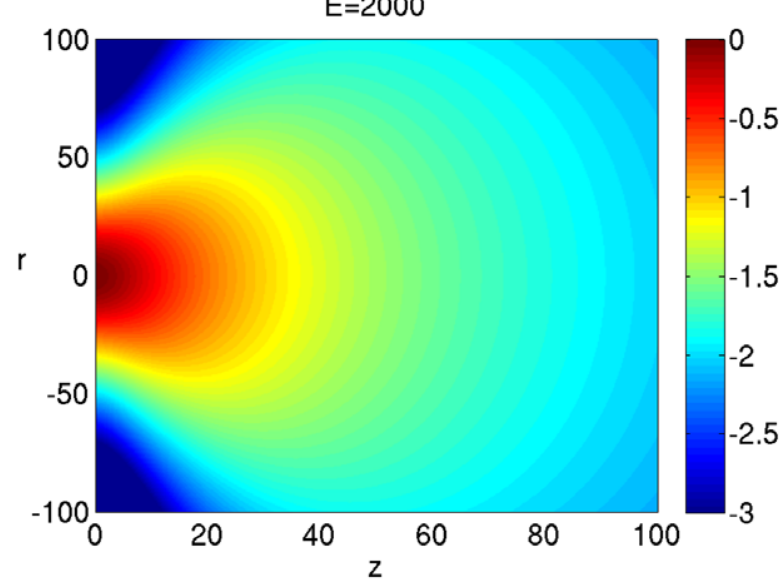

Figure 22. Generalized solution. Two-dimensional profiles of the density in $(z, r)$, for $u_{\theta} \neq 0, D=-10, F=1$, and $E=0,500,1000$, 2000 , in logarithmic scale.

Clearly, from equation (10), the value of $E$ determines the magnitude of the azimuthal velocity. We plot in figure 21 the ratio between the total velocity $v=\left(u_{r}^{2}+u_{z}^{2}+u_{\theta}^{2}\right)^{1 / 2}$ and the azimuthal velocity $u_{\theta}$, at the injection boundary $z=0$, as a function of $r$, for different values of $E$. Note that $u_{\theta}$ increases linearly with $r$, and $v / u_{\theta}$ does not depend on $D$. The inclusion of a non-null azimuthal velocity at injection causes a distortion of the density profile. Figure 22 shows the two-dimensional density profile in $(z, r)$, for $E=0,500,1000,2000, D=-10$, and $F=1$. The notable effect of the azimuthal velocity is that the density decreases much more sharply along the axial direction, when $u_{\theta}$ is sufficiently large. This effect is even more evident when $D>0$, i.e. when the plume is bounded by a propagation cone. Figure 23 shows the two-dimensional density profile in $(z, r)$ for $D=3$ and $F=1$ (i.e. for the PK solution). Clearly, the cone of propagation is distorted and the isocontour $n=0$ becomes more and more curved, with increasing $E$.

\section{Conclusions}

We have analyzed in detail the self-similar solutions presented in [1-3], that approximately resolve a fluid model for the steady-state, axisymmetric expansion of an electrostatic, quasi-neutral, collisionless plasma plume. It is important to recall that the semi-analytical solutions can be computed in a negligible fraction of the time needed to obtain a full numerical solution, and they are often preferable, as long as we have a measure for the errors. Therefore, in order to judge whether such approximate solutions can reasonably be used in real applications, we have numerically solved the underlying model and we have calculated errors between the approximate and the numerical solutions. A common characteristic of all the solutions is that the errors decrease with increasing axial injection velocity $u_{c}$. For the PK [1] and AF [3] solutions, the errors are smaller than $1 \%$ for $u_{c} \gtrsim 20$. The KT solution yields larger errors. Each solution is characterized by a given profile for the density and velocity at injection, and the user has little freedom for adjusting the profiles for more general situations. For this reason, we have introduced a new class of solutions, that includes the PK and the KT solutions as special cases. We have shown that this new family of solutions can describe fairly general injection profiles, yielding errors comparable to the PK and AF models. Finally, we have shown that a simple extension to the case of a non-null azimuthal velocity $u_{\theta}$ is possible. Interestingly, the effect of the azimuthal velocity is to distort the geometry of the plume in such a way that the density decreases more sharply in the vicinity of the 

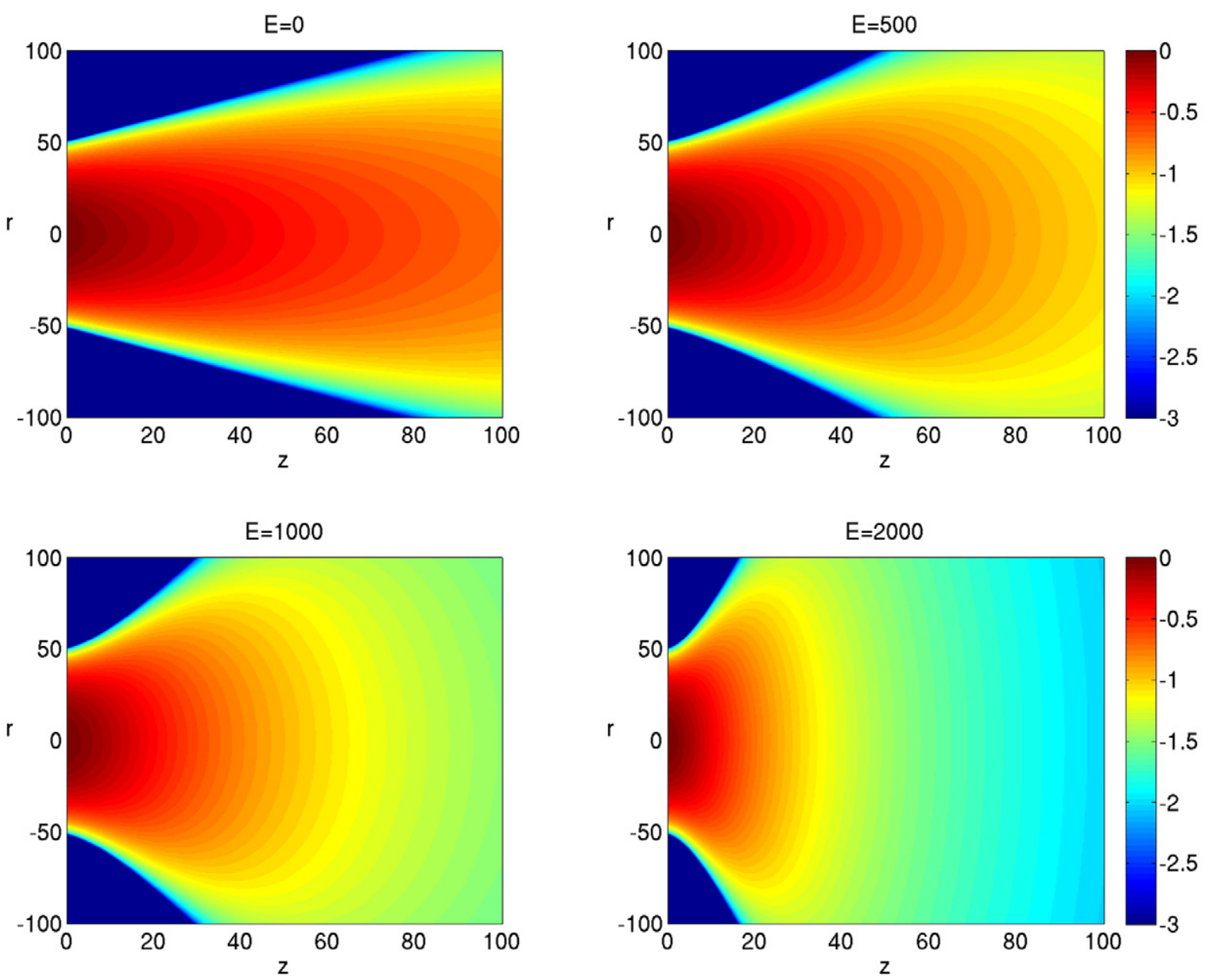

Figure 23. Generalized solution. Two-dimensional profiles of the density in $(z, r)$, for $u_{\theta} \neq 0, D=3, F=1$, and $E=0,500,1000,2000$, in logarithmic scale.

injection boundary. In real applications, such a scenario might be more favorable for plasma contactors, where it is preferable to have the plume as close as possible to its source, in order to maximize the efficiency of the charge neutralization process.

\section{Acknowledgments}

We thank G L Delzanno, J E Borovsky and M F Thomsen for useful discussions. This work was partially funded by the Laboratory Directed Research and Development program (LDRD), under the auspices of the National Nuclear Security Administration of the US Department of Energy by Los Alamos National Laboratory, operated by Los Alamos National Security LLC under contract DE-AC52-06NA25396.

\section{Appendix. Derivation of equations (12)-(17)}

Substituting equations (7)-(9) in the continuity equation (1) yields:

$$
2 n_{c} u_{c} a^{\prime}+a\left(u_{c} n_{c}^{\prime}+n_{c} u_{c}^{\prime}\right)=0,
$$

which reduces to

$$
\left(a^{2} n_{c} u_{c}\right)^{\prime}=0
$$

from which equation (12) follows. For what concerns the momentum equation in the $r$ direction (2) one gets

$$
\frac{\gamma\left(n_{c} n_{t}\right)^{\gamma} n_{t}^{\prime}}{n_{c} n_{t}^{2}}+\eta a u_{c} u_{t}^{2}\left(a^{\prime} u_{c}^{\prime}+u_{c} a^{\prime \prime}\right)=0,
$$

that can be separated as

$$
\frac{u_{c} a}{n_{c}^{\gamma-1}}\left(u_{c} a^{\prime}\right)^{\prime}=-\frac{\gamma n_{t}^{\gamma-2} n_{t}^{\prime}}{\eta u_{t}^{2}}=\gamma C,
$$

from which equations (15) and (17) follow.

Substituting equations (7)-(9) in the momentum equation in the $z$ direction (3) one obtains

$$
\gamma\left(n_{c} n_{t}\right)^{\gamma-2}\left(n_{t} n_{c}^{\prime}-\frac{a^{\prime}}{a} \eta n_{c} n_{t}^{\prime}\right)+u_{c} u_{t}^{2} u_{c}^{\prime}=0
$$

which is unfortunately not separable, and this is the source of the over-determination of the system of equations (12)-(17). In order to make equation (60) separable, one has to make the assumption

$$
\gamma n_{c}^{\gamma-2} n_{c}^{\prime}+u_{c} u_{c}^{\prime}=0
$$

that can be integrated to obtain equation (13). Finally, using this assumption and taking the $\eta$ derivative of equation (60), 
the result separates as

$$
\frac{n_{c}^{\prime} a}{n_{c} a^{\prime}}=-\frac{2 n_{t}^{\prime} u_{t}}{2 n_{t} u_{t}^{\prime}-(\gamma-1) n_{t}^{\prime} u_{t}}=D
$$

that ultimately yields, by integration, equations (14) and (16).

\section{References}

[1] Parks D E and Katz I 1979 A preliminary model of ion beam neutralization 14th Int. Electric Propulsion Conf. (Princeton, NJ)

[2] Korsun A G and Tverdokhlebova E M 1997 The characteristics of the EP exhaust plume in space 33rd Joint Prop. Conf. (Seattle, WA) AIAA-97-3065

[3] Ashkenazy J and Fruchtman A 2001 Plasma plume far field analysis 27th Int. Electric Propulsion Conf. (Pasadena, CA)

[4] Boyd I D and Yim J T 2004 Modeling of the near field plume of a Hall thruster J. Appl. Phys. 95 4575-84

[5] Zhong Q, Pingyang W, Zhaohui D and Xiaolu K 2008 Study of plume characteristics of a stationary plasma thruster Plasma Sci. Technol. 10612

[6] Yan L, Wang P-Y, Ou Y-H and Kang X-L 2012 Numerical study of Hall thruster plume and sputtering erosion J. Appl. Math. 2012327021

[7] Dannenmayer K and Mazouffre S 2013 Electron flow properties in the far-field plume of a hall thruster Plasma Sources Sci. Technol. 22035004

[8] Wilbur P J and Laupa T G 1988 Plasma contactor design for electrodynamic tether applications Adv. Space Res. 8 221-4

[9] Sanmartin J R, Lorenzini E C and Martinez-Sanchez M 2010 Electrodynamic tether applications and constraints $J$. Spacecr. Rockets 47 442-56

[10] Crow J E, Auer P L and Allen J E 1975 The expansion of a plasma into a vacuum J. Plasma Phys. 14 65-76

[11] Mora P 2003 Plasma expansion into a vacuum Phys. Rev. Lett. 90185002

[12] Taccogna F, Longo S and Capitelli M 2002 Particle-in-cell with Monte Carlo simulation of SPT-100 exhaust plumes J. Spacecr. Rockets 39 409-19
[13] Boyd I D and Dressler R A 2002 Far field modeling of the plasma plume of a Hall thruster J. Appl. Phys. 92 1764-74

[14] Garrigues L, Bareilles J, Boeuf J P and Boyd I D 2002 Modeling of the plasma jet of a stationary plasma thruster $J$. Appl. Phys. 91 9521-8

[15] Taccogna F, Longo S and Capitelli M 2004 Very-near-field plume simulation of a stationary plasma thruster Eur. Phys. J. Appl. Phys. 28 113-22

[16] Boyd I D 2006 Numerical simulation of hall thruster plasma plumes in space IEEE Trans. Plasma Sci. 34 2140-7

[17] Taccogna F, Longo S, Capitelli M and Schneider R 2007 Particle-in-cell simulation of stationary plasma thruster Contrib. Plasma Phys. 47 635-56

[18] Kronhaus I, Kapulkin A, Guelman M and Natan B 2012 Investigation of two discharge configurations in the CAMILA Hall thruster by the particle-in-cell method Plasma Sources Sci. Technol. 21035005

[19] Korsun A G, Tverdokhlebova E M and Gabdullin F F 2004 Mathematical model of hypersonic plasma flows expanding in vacuum Comput. Phys. Commun. 164 434-41

[20] Gabdullin F F, Korsun A G and Tverdokhlebova E M 2008 The plasma plume emitted onboard the international space station under the effect of the geomagnetic field IEEE Trans. Plasma Sci. 36 2207-13

[21] Peraire J and Martinez-Sanchez M 2006 Development of advanced hall plume models Technical Report DTIC Document

[22] Bluman G W and Cole J D 1974 Similarity Methods for Differential Equations (Berlin: Springer)

[23] Burm K T A L, Goedheer W J and Schram D C 1999 The isentropic exponent in plasmas Phys. Plasmas 6 2622-7

[24] Taccogna F, Pagano D, Scortecci F and Garulli A 2014 Three-dimensional plume simulation of multi-channel thruster configuration Plasma Sources Sci. Technol. 23065034

[25] Merino M, Ahedo E, Bombardelli C, Urrutxua H and Pelaez J 2011 Hypersonic plasma plume expansion in space 32nd Int. Electric Propulsion Conf. (Wiesbaden, Germany)

[26] Kelley C T 2003 Solving Nonlinear Equations with Newton's Method vol 1 (Philadelphia, PA: SIAM) 